C-A/AP/\#115

October 2003

Booster Fault Study No. 17:

Proton Beam on the D6 Septum Magnet

K.A. Brown, C.J. Gardner

Brookhaven National Laboratory

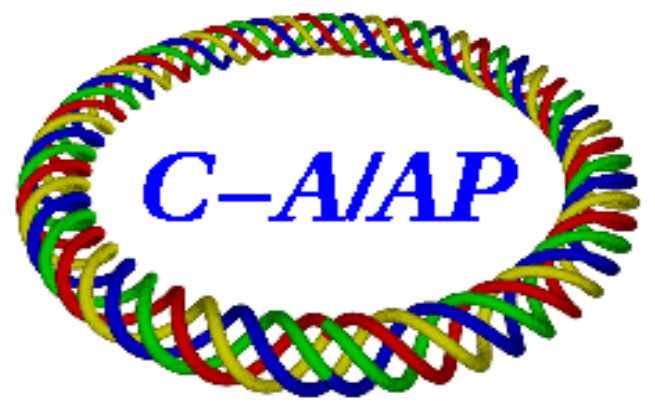

Collider-Accelerator Department Brookhaven National Laboratory

Upton, NY 11973 


\title{
Booster Fault Study No. 17: Proton Beam on the D6 Septum Magnet
}

\author{
K.A. Brown and C.J. Gardner
}

October 8, 2003

Following is a report on the Booster fault study carried out 30 January 2003. The original fault study plan is reproduced here followed by the actual study report.

\section{Study Plan}

\subsection{Goal}

The goal of this study is to produce a primary beam loss on the D6 septum magnet and measure the resulting prompt radiation in the Booster-NSRL penetration region. Measurements are to be made on the berm over the region and in the NSRL tunnel near the penetration headwall and at the "stub" gate. The study is to be conducted in accordance with AGS OPM 9.1.9.

\subsection{Initial (non-fault) Beam Conditions}

1. The study is to be conducted with deuterons from Tandem or with protons from Linac.

2. The maximum kinetic energy is $1.0 \mathrm{GeV}$ per nucleon for deuterons and $2.0 \mathrm{GeV}$ for protons. The repetition period is at least 4 seconds.

3. The maximum intensity is $4 \times 10^{10}$ deuterons or $4 \times 10^{11}$ protons per Booster cycle. 


\subsection{Method}

1. Establish beam on a Booster cycle with flat top at the desired energy. Record flat top energy and repetition period.

2. The Booster RF should be turned off just after beam reaches flat top energy. Then, as the field increases (slightly) at the end of the flat top, any surviving beam will be lost on the radially inward side of the vacuum chamber. The B6 dump bump should be adjusted so that this beam is lost on the B6 dump.

3. Establish desired intensity with no loss during flat top. Record beam current transformer trace and ring loss monitor output throughout the Booster cycle.

4. Using the flat trim windings on the C7, D1, D4, D7 and E1 dipoles, make a local distortion (bump) of the equilibrium orbit at the D6 septum magnet during the flat top portion of the magnetic cycle.

5. Adjust the amplitude of the bump so that all beam is lost on the D6 septum. A radial shift to the outside may be required.

6. Record beam current transformer trace and ring loss monitor output for the loss condition. Record output from Booster chipmunks NM134 and NM133. Record Radius and Bump parameters.

7. Measure radiation levels on the Booster berm and in the NSRL tunnel under the loss condition.

\subsection{Survey Locations}

Figure 1 is a schematic of the Booster-NSRL penetration region.

Figure 2 shows the actual plan view of the region. A cross-section of the berm in the area of thin soil cover is shown in Figure 3. The nominal cross-section of the Booster tunnel and berm is shown in Figure 4. Refering to these figures, the survey locations are:

1. On Booster berm directly over the D6 septum magnet. The vent shown in Figures 1 and 2 can be used as a reference point to locate the point on the berm over the D6 septum magnet. The distance between this point and the vent is some 80 feet. 
2. On the berm in the area of thin soil cover indicated in Figure 1.

3. Inside the NSRL tunnel near the penetration headwall and at the stub gate indicated in Figure 1.

All surveys are to be conducted with the HP1010 meter unless otherwise noted.

\subsection{Radiation Estimates}

To obtain an estimate of the radiation levels we assume a point loss directly under the areas of interest and apply the formula

$$
H=\frac{1}{r^{2}}\left(1.6 \times 10^{-6}\right) L e^{-d / 117}
$$

given by Tesch [1]. Here $H$ is the dose rate (mrem/hour) on the berm directly above the loss point, $L$ is the loss rate $(\mathrm{GeV} / \mathrm{s}), d$ is the mass-density thickness $\left(\mathrm{g} / \mathrm{cm}^{2}\right)$ of the berm shielding, and $r$ is the distance (meters) from the loss point.

\subsubsection{On the Berm Directly over the D6 Magnet}

On the berm directly over the D6 septum magnet, the depth of the soil cover is at least $427 \mathrm{~cm}$ (14 feet) and the distance from the magnet is $r=5.49 \mathrm{~m}$ (18 feet). Taking the density of the soil to be $1.8 \mathrm{~g} / \mathrm{cm}^{3}$, we have $d=427 \times 1.8=769 \mathrm{~g} / \mathrm{cm}^{2}$. For a point loss of $10^{11}$ protons per second at $2 \mathrm{GeV}$ kinetic energy, the dose rate on the berm would then be $15 \mathrm{mrem} /$ hour. For a point loss of $10^{10}$ deuterons per second at $1 \mathrm{GeV}$ kinetic energy per nucleon, the dose rate would be $1.5 \mathrm{mrem} / \mathrm{hour}$.

\subsubsection{On the Berm in the Area of Thin Soil Cover}

Even though the loss we are considering occurs on the D6 magnet, let us assume that the loss occurs directly under the area of thin soil cover. Here the depth of the soil may be as little as $366 \mathrm{~cm}$ (12 feet) and the distance from the loss point may be as little as $r=5.18 \mathrm{~m}$ (17 feet). Taking the density of the soil to be $1.8 \mathrm{~g} / \mathrm{cm}^{3}$, we have $d=366 \times 1.8=659 \mathrm{~g} / \mathrm{cm}^{2}$. For a point loss of $10^{11}$ protons per second at $2 \mathrm{GeV}$ kinetic energy, the

dose rate on the berm would then be $43 \mathrm{mr} /$ hour. For a point loss of $10^{10}$ 
deuterons per second at $1 \mathrm{GeV}$ kinetic energy per nucleon, the dose rate would be $4.3 \mathrm{mr} /$ hour. These are upper limits on the expected dose rates in the area of thin soil cover. (During the Summer of 2003 the berm in the Booster-NSRL penetration region was rebuilt and stabilized with a retaining wall. The soil cover is now at least 14 feet in this region.)

\subsubsection{At the Penetration Headwall}

Here, in a fault study [2] carried out on 9 December 2000, a dose rate of 7.5 mrem per hour was measured by Chipmunk NM066 which was positioned at the headwall close to the present position of Chipmunk NM134. The beam loss in this case was $1.1 \times 10^{12}$ protons per second on the D6 dump at a kinetic energy of $1.74 \mathrm{GeV}$. (The D6 dump has since been removed and replaced with the D6 septum magnet.) Based on this measurement, the expected dose rate at the headwall for a loss on the D6 septum magnet of $10^{11}$ protons per second at $2 \mathrm{GeV}$ kinetic energy would be 0.78 mrem per hour. For a loss on the septum magnet of $10^{10}$ deuterons per second at $1 \mathrm{GeV}$ kinetic energy per nucleon, the dose rate would be a factor of ten smaller.

\subsubsection{At the Stub Gate}

Here the dose rate for a loss on the D6 magnet of $10^{10}$ deuterons per second at $1 \mathrm{GeV}$ kinetic energy per nucleon has been estimated by Adam Rusek to be 0.009 mrem per hour.

\section{Actual Study}

\subsection{Conditions}

Protons from the polarized proton source (and Linac) were injected into Booster and accelerated to a kinetic energy of $1.5 \mathrm{GeV}$. Although the study plan called for a magnetic cycle with a flat top, it was more convenient to use the standard polarized proton magnetic cycle.

The magnetic cycle repetition period was $324 / 60=5.4$ seconds.

Under fault conditions all beam loss occurred approximately $108 \mathrm{~ms}$ from BT0 (Booster T zero) where the field was 5115 Gauss. This gives a rigidity of $7.092 \mathrm{Tm}$ (assuming radius of curvature $\rho=13.8656$ meters in the 
Booster dipoles) and a kinetic energy of $1.4 \mathrm{GeV}$. The loss was $2.4 \times 10^{11}$ protons per Booster cycle. With a repetition period of 5.4 seconds, this gives a loss rate of $4.4 \times 10^{10}$ protons per second.

\subsection{Loss Setup}

The currents in the flat trim windings on dipoles C7, D1, D4, D7 and E1 were programmed to produce a local loss on the D6 septum magnet. This is shown in Figure 5. Here the three oscilliscope traces are the normalized beam current, the Booster main magnet current, and the current in the flat trim winding on dipole D1. The D1 trim winding current increases from 0 to $+500 \mathrm{~A}$ in $10 \mathrm{~ms}$ starting $100 \mathrm{~ms}$ from BT0. It then decreases back to 0 in the next $10 \mathrm{~ms}$. The currents in the C7, D4, D7, and E1 trim windings had the same time dependence, but with maximum amplitudes $-120,+300,+500$, and +120 A respectively. All of the beam is lost as the currents increase to their maximum amplitudes. The time of the loss is approximately $108 \mathrm{~ms}$ from BT0.

\subsection{Loss Measurements}

Beam loss was measured with the circulating beam current transformer and with the RLM (Ring Loss Monitor) system. The loss rate determined from the beam current transformer was $4.4 \times 10^{10}$ protons per second as discussed above. At a kinetic energy of $1.4 \mathrm{GeV}$, this gives an energy loss of $6.2 \times 10^{10} \mathrm{GeV}$ per second.

The RLM readings under the fault condition are listed in Table 1. Here the window of observation was from 100 to $120 \mathrm{~ms}$ from BT0 which brackets the time when beam is lost. The readings show that most of the loss occurs between the D3 and E3 straight sections with the maximum loss centered on the D6 septum magnet and the D7 dipole. 
Table 1: Booster RLM Readings under Fault Condition

\begin{tabular}{|c|c|c|c|}
\hline RLM & Counts & RLM & Counts \\
\hline D1 & 37 & F1 & 193 \\
D2 & 74 & F2 & 185 \\
D3 & 112 & F3 & 162 \\
D4 & 2289 & F4 & 332 \\
D5 & 2196 & F5 & 75 \\
D6 & 4014 & F6 & 1738 \\
D7 & 4094 & F7 & 1801 \\
D8 & 2855 & F8 & 192 \\
\hline E1 & 2251 & A1 & 94 \\
E2 & 2170 & A2 & 162 \\
E3 & 424 & A3 & 23 \\
E4 & 18 & A4 & 80 \\
E5 & 203 & C5 & 121 \\
E6 & 734 & C6 & 188 \\
E7 & 496 & C7 & 215 \\
E8 & 241 & C8 & 98 \\
\hline
\end{tabular}

\subsection{Radiation Measurements}

Radiation measurements were made with the HP1010 meter and the E600 "rem ball". The levels were also monitored by Chipmunks NM133 and NM134. The positions of the chipmunks are shown in Figure 1. NM134 is mounted on the stub tunnel headwall near the beampipe. NM133 is mounted on the wall near the stub tunnel gate.

\subsubsection{On the Berm over the D6 Septum Magnet}

Here the HP1010 meter and E600 rem ball were placed on the ground and gave readings that are summarized in Table 2 . The measured dose rate due to the fault is 0.112 mrem per hour. For a point loss of $10^{11}$ protons per second at $2 \mathrm{GeV}$ kinetic energy, the predicted dose rate is 15 mrem/hour. The predicted dose rate for a point loss of $4.4 \times 10^{10}$ protons per second at $1.4 \mathrm{GeV}$ kinetic energy is then $1.4 \times 4.4 \times 15 / 20=4.6 \mathrm{mrem}$ per hour. This is 41 times higher than the observed dose rate. 
Table 2: Radiation Levels on the Berm over the D6 Septum Magnet

\begin{tabular}{|c|c|c|}
\hline Condition & Meter & $\begin{array}{c}\text { Dose Rate } \\
(\mathrm{mrem} / \mathrm{h})\end{array}$ \\
\hline Fault & HP1010 & $<0.05$ \\
& E600 & 0.183 \\
\hline Beam Off & HP1010 & $<0.05$ \\
& E600 & 0.071 \\
\hline
\end{tabular}

\subsubsection{In the Stub Tunnel and at the Stub Gate}

Here the E600 rem ball was placed on the floor in the stub tunnel downstream of the headwall and upstream of the concrete blocks shown in Figure 1. The HP1010 meter was held by a technician standing at the stub gate. The readings from both devices are summarized in Table $\mathbf{3}$.

Table 3: Radiation Levels in the Stub Tunnel and at the Stub Gate

\begin{tabular}{|c|c|c|c|}
\hline Condition & Location & $\underline{\text { Meter }}$ & $\frac{\text { Dose Rate }}{(\mathrm{mrem} / \mathrm{h})}$ \\
\hline Fault & Tunnel & E600 & 0.425 \\
& Gate & HP1010 & 0.05 \\
\hline Beam Off & Tunnel & E600 & 0.062 \\
& Gate & HP1010 & 0.05 \\
\hline
\end{tabular}

Here we see that the dose rate in the stub tunnel due to the fault is 0.363 mrem per hour. At the gate the HP1010 meter registered no discernable difference between the beam off and fault conditions.

As discussed in Section 1.5.3, the expected dose rate at the headwall for a loss on the D6 septum magnet of $10^{11}$ protons per second at $2 \mathrm{GeV}$ kinetic energy is 0.78 mrem per hour. The expected dose rate for a loss of $4.4 \times 10^{10}$ protons per second at $1.4 \mathrm{GeV}$ kinetic energy is then $0.24 \mathrm{mrem}$ per hour. This is in good agreement with the observed rate of $0.363 \mathrm{mrem}$ per hour. 


\subsubsection{Chipmunks}

Chipmunk NM134 (mounted on the headwall near the beampipe) registered a dose rate of 0.35 mrem per hour due to the fault. This is in good agreement with the E600 rem ball measurements in Table 3.

Chipmunk NM133 (mounted on the wall near the gate) registered a dose rate of 0.15 mrem per hour due to the fault.

\section{References}

[1] K. Tesch, Health Physics, Vol. 44, No. 1, pp. 79-82, January, 1983.

[2] C. J. Gardner, "Booster Fault Study for the BAF Penetration Site", AGS Studies Report 375, January 13, 2000. 


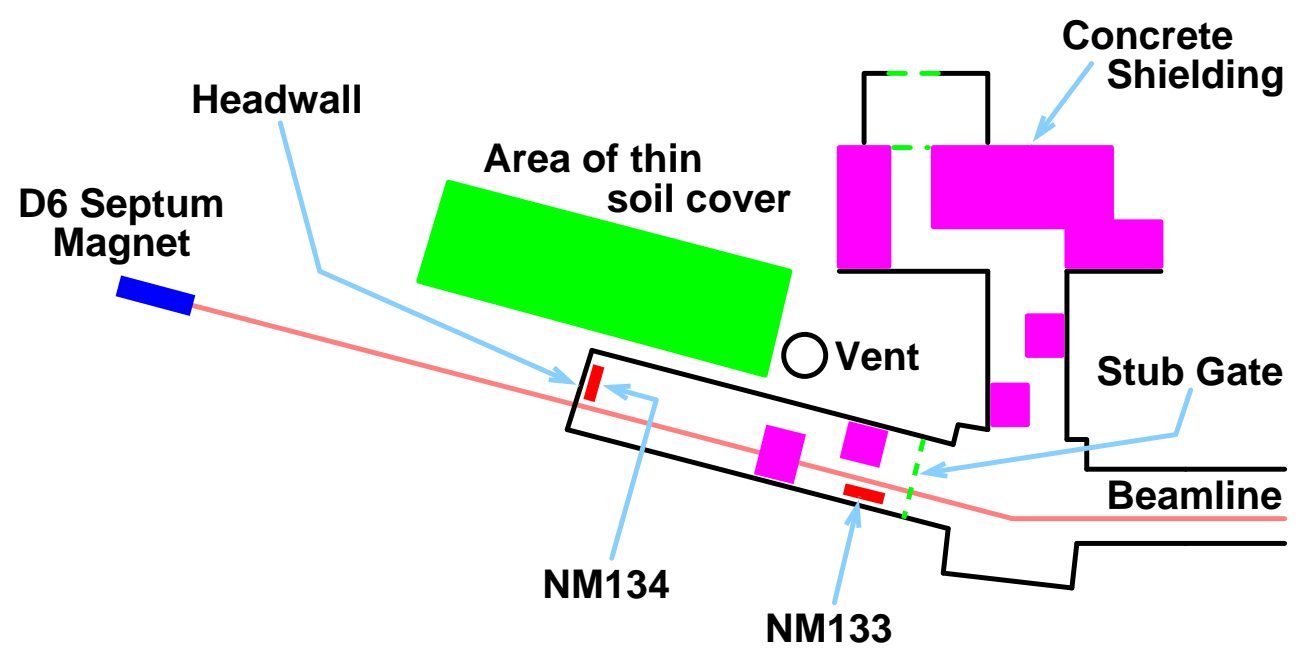

Figure 1: Schematic of the Booster-NSRL penetration region. The stub tunnel is the area between the headwall and the stub gate. Radiation measurements are to be taken on top of the berm directly over the D6 septum magnet and on the side of the berm in the area of thin soil cover. Measurements also are to be taken in the tunnel near the headwall and at the stub gate. 


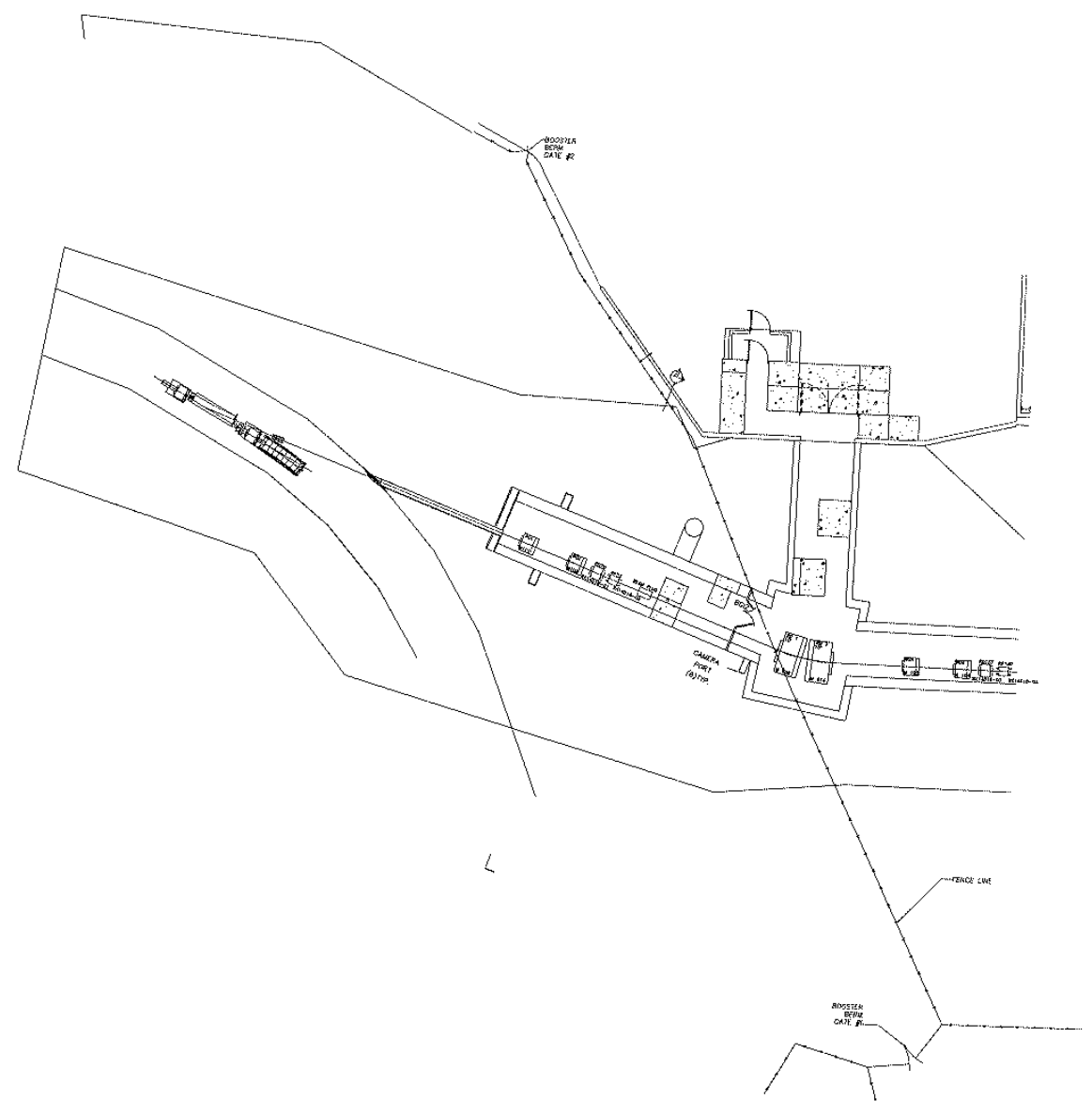

Figure 2: Plan view of Booster-NSRL penetration region. This is from drawing number 82000002 . 


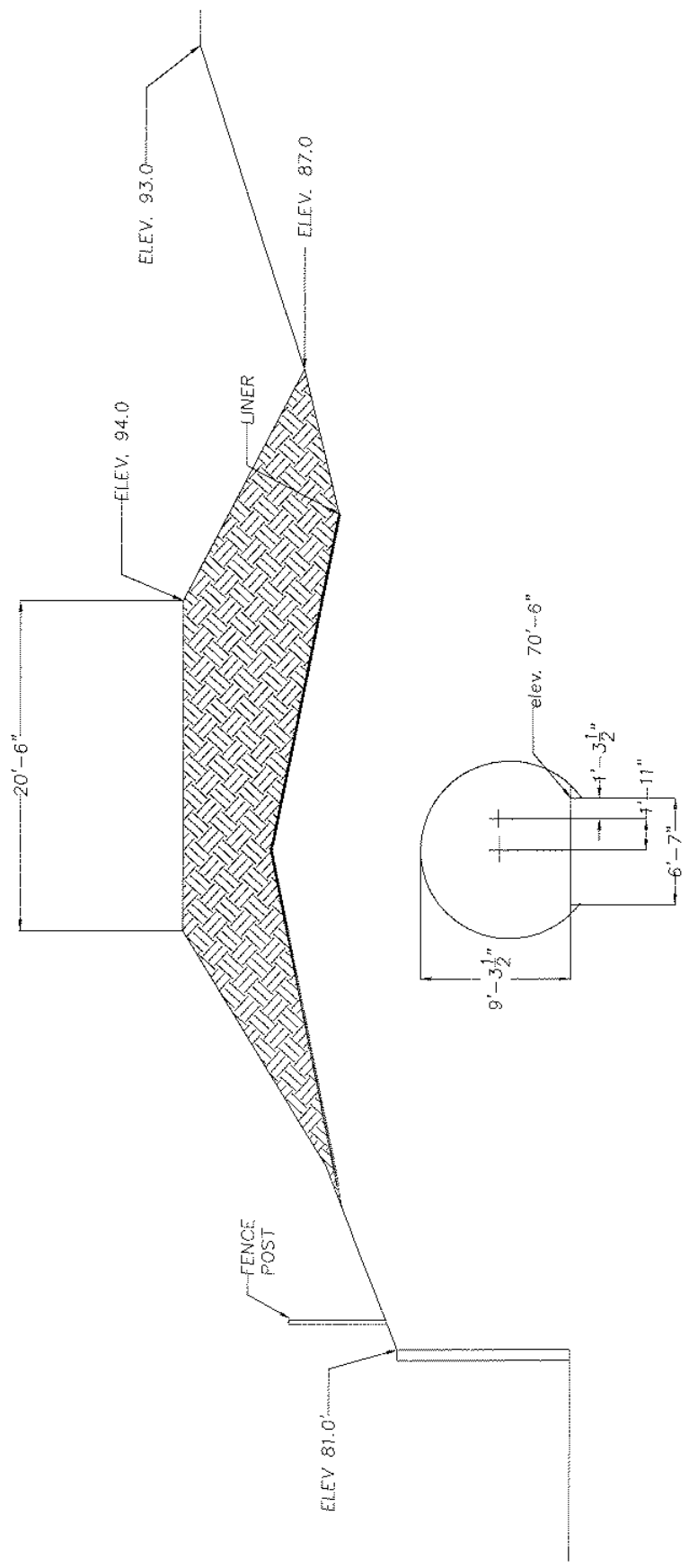

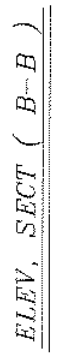

Figure 3: Cross-section of berm in the area of thin soil cover. This is crosssection B from drawing number 82000002. 


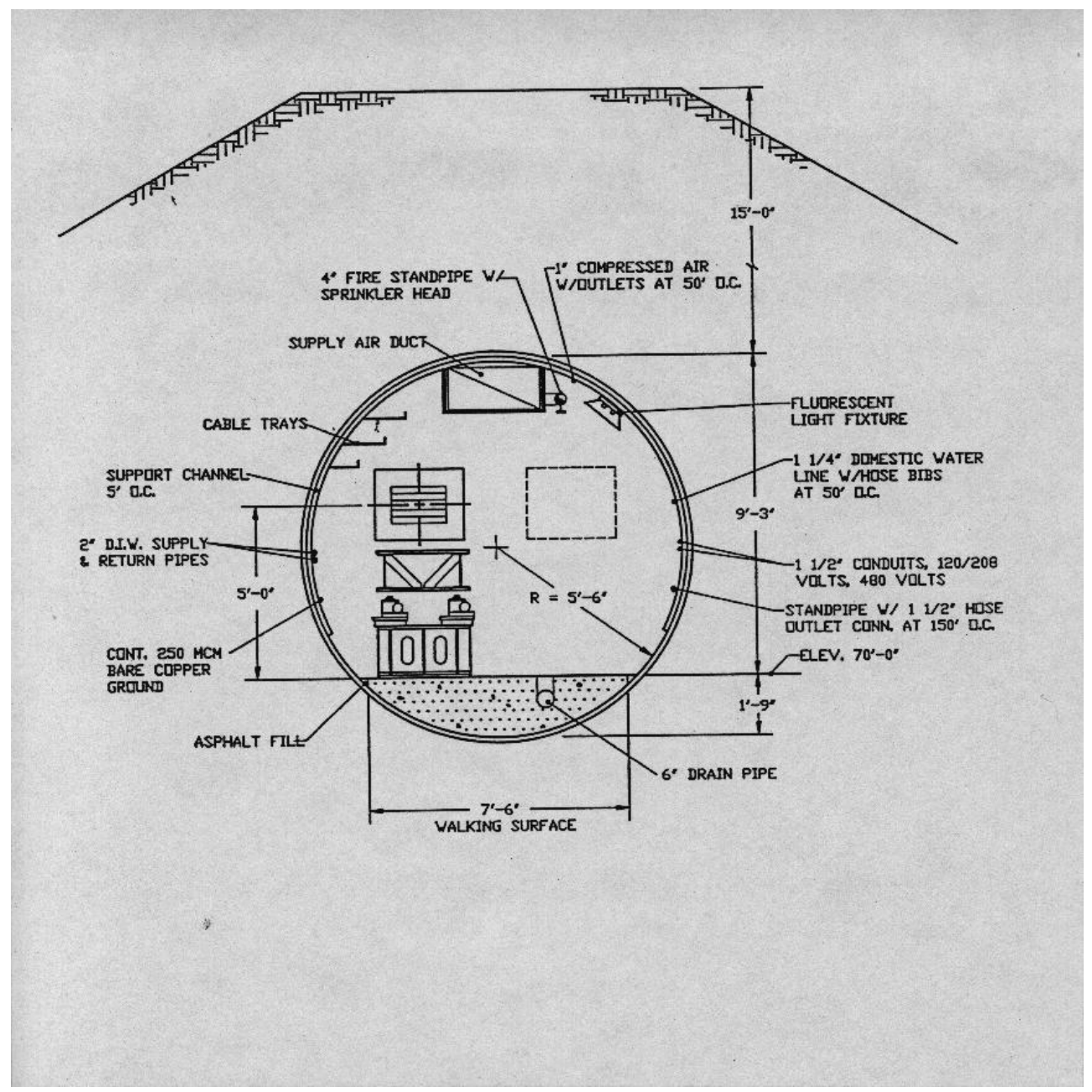

Figure 4: Nominal cross-section of Booster tunnel and berm. This is from the Ags Booster Final Safety Analysis Report, February 27, 1991. Note that the elevation of the tunnel floor is 70 feet. The center of the beam pipe is 5 feet above the floor and the top of the tunnel is 9 feet 3 inches above the floor. The nominal depth of soil over the tunnel is 15 feet. 


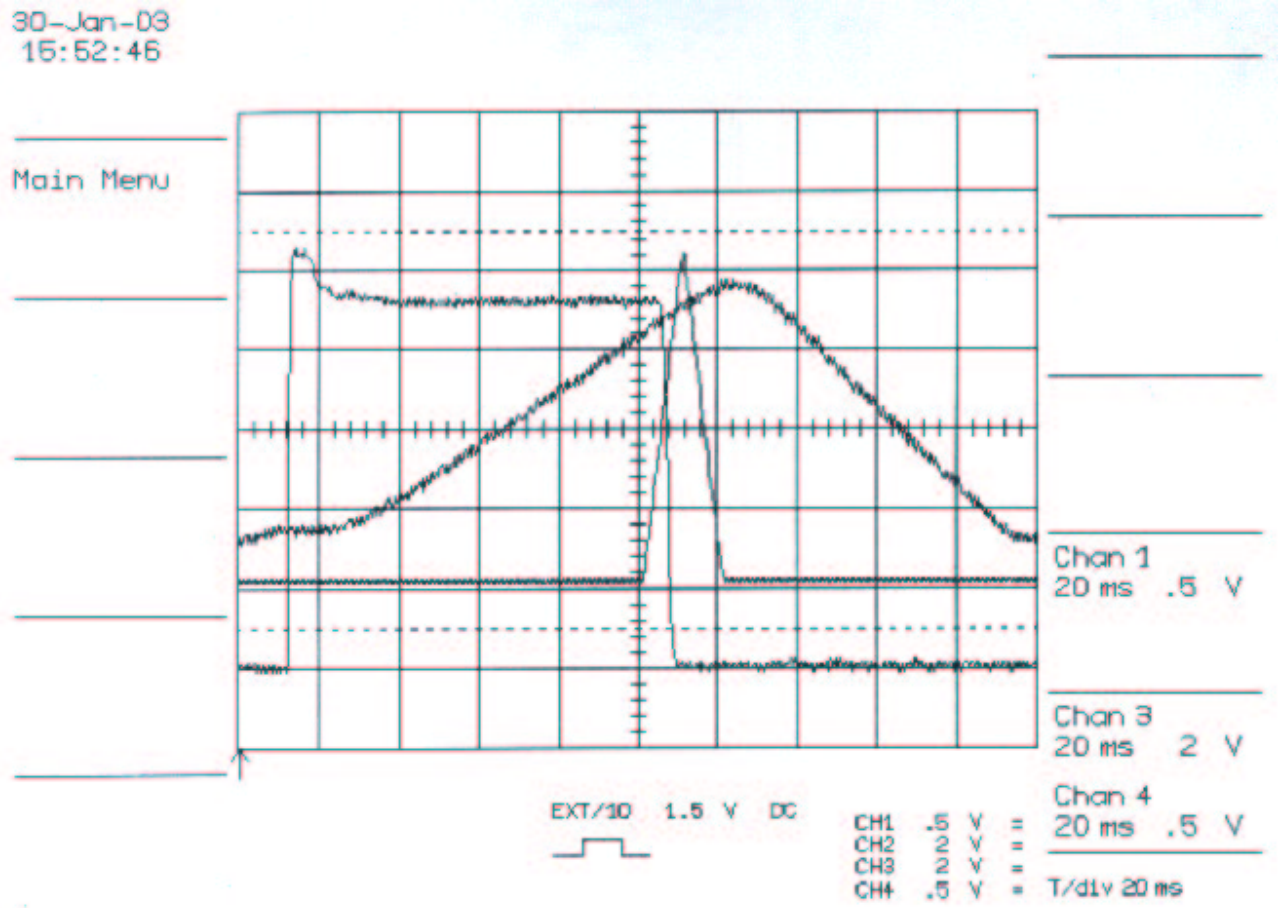

Figure 5: Fault study loss setup. Here the three oscilliscope traces are the normalized beam current, the Booster main magnet current, and the current in the flat trim winding on dipole D1. The trim winding current increases from 0 to $500 \mathrm{~A}$ in $10 \mathrm{~ms}$ starting $100 \mathrm{~ms}$ from BT0. It then decreases back to 0 in the next $10 \mathrm{~ms}$. All of the beam is lost as the current increases to $500 \mathrm{~A}$. The loss time is approximately $108 \mathrm{~ms}$ from BT0. 